Tagung „Homo medialis“

Homo medialis.

Perspektiven und Probleme einer Anthropologie der Medien

Bericht über die IZMM-Tagung am 8. und 9. November 2002

\section{MANFred L. PIRner}

\section{Medienanthropologie? Ausgangs- punkte und Fragestellungen der Tagung}

Angesichts der rasant zunehmenden Bedeutung der elektronischen Medien für Individuum und Gesellschaft stellt sich verschärft die Frage nach grundlegenden, tragfähigen und für verantwortungsvolles Handeln hilfreichen Deutungen des Verhältnisses von Mensch und Medium.

Entwickeln einerseits medieneuphorische Stimmen bereits die Vision der Perfektionierung des Menschen durch die Medien im Sinne einer Überwindung seiner leiblichen Hinfälligkeit und Sterblichkeit, so warnen andererseits medienkritische Geister vor der technizistischen Degradierung und Verdrängung des genuin Menschlichen.

Was machen die Medien mit dem Menschen und

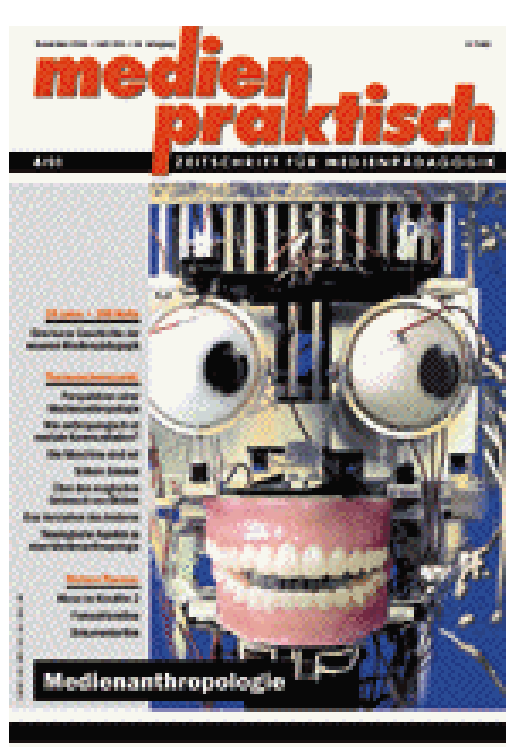

Themenheft „Medienanthropologie“ der Zeitschrift „medien praktisch" Ausgabe 4/ 2001 was macht der Mensch mit den Medien? Diese beiden typischen Fragen der Medienforschung lassen sich zuspitzen zur Frage: Inwiefern machen Medien den Menschen erst zum Menschen oder inwiefern beeinträchtigen sie sein Menschsein?

Im Sinne dieser Fragestellungen hat sich die erfreulich interdisziplinäre wissenschaftliche Beschäftigung mit den Medien in jüngerer Zeit verstärkt ethnologischen, kulturanthropologischen, kultur- und religionssoziologischen Ansätzen zugewandt und von sym- bol-, ritual-, mythos- und religionstheoretischen Sichtweisen profitiert.

Den Ausgangspunkt der Tagung bildete dementsprechend die Frage, ob die gegenwärtigen anthropologischen Tendenzen in weiten Teilen der Medienforschung nicht zu einer "Anthropologie der Medien" zusammengefasst werden können und welche neuen Perspektiven auf die Medien sich möglicherweise aus einer programmatischen Rückbesinnung auf anthropologische $\mathrm{G}$ rundfragen und - themen gewinnen lassen.

\section{Medienanthropologie! Beiträge und Aspekte}

D r. Manfred Fassler, Professor für Kulturanthropologie und europäische Ethnologie an der Universität Frankfurt, eröffnete die Tagungsvorträge mit seinem Beitrag "Die mediale Selbstbefähigung des Menschen". Wie der Titel bereits andeutet, stellen sich für Fassler in der beginnenden Systematisierung des Bild-, Zeichenund Zahlensinnes und deren In-Dienstnahme für ein entstehendes $\mathrm{Ge}$ schichts-Denken und für eine entstehende Philosophie allgemein menschliche Notwendigkeiten und Fähigkeiten dar. Diese Notwendigkeiten bestehen darin, die andauernden sinnlichen Eindrücke in lebensund überlebensorientiertes Gruppenverhalten zu übersetzen, also zu signalisieren, zu kommunizieren, zu koordinieren, zu kultivieren. Näherhin besteht eine solche Kultivierung dann darin, Zeichen-Kulturen 1. Ordnung in solche der 2. Ordnung zu überführen, in Sprachen der Selbstbeobachtung, der Selbstdarstellung, des Selbstentwurfes. Der Mensch muss sich gleichsam in seinen Darstellungen selbst erfinden, um das erhalten zu können, was wir Bewusstsein nennen.

Von diesen Grundlagen her bestimmt Fassler sein Konzept einer Medienanthropolo- 
gie: Sie sucht Wege, die Entwicklungen in der Selbstorganisation unterschiedlicher menschlicher Kulturen zu beschreiben. Sie tut dies, wie Fassler betont, nicht aus der falschen $\mathrm{G}$ ewissheit heraus, im Menschen seien die medialen Fähigkeiten enthalten, sondern sie tut dies aus dem Verständnis heraus, dass sich der Mensch durch die von ihm erfundenen, entwickelten, eingesetzten und gebrauchten komplexen Systeme stets veränderte Anthropologien erzeugt. Dies gilt auch für das Konzept der Medienanthropologie.

Jedenfalls aber hält Fassler eine solchermaßen kulturanthropologisch sich verstehende Medienanthropologie für so grundlegend, dass er dafür plädiert, sie als eigenständige wissenschaftliche Disziplin zu etablieren.

Als freier Medien- und PR-Berater brachte der Politikwissenschaftler D r. Christof E hrhart (Schweiz) die Perspektive der „harten” ökonomischen Medienwelt ein: Unter der Überschrift ${ }_{\text {„H}} \mathrm{H}$ andlungsk alküle im M edienfeld. Rollenmodelle des medialen M enschen" stellte er Modelle der unterschiedlichen Medien-Berufe bzw. Rollenfunktionen in der Medienwirtschaft vor, zu deren $\mathrm{Be}$ schreibung nur wenige grundlegend-elementare Kategorien herausgearbeitet werden.

Prof. D r. Klaas H uzing hat nicht nur den Lehrstuhl für (evangelische) Systematische Theologie an der Universität Würzburg inne, sondern ist zugleich anerkannter Romanautor und als solcher Mitglied im PENClub. Im zweiten Band seiner auf drei Bände angelegten "Ästhetischen Theologie" beschäftigt er sich - nach einer literarischen Anthropologie im ersten Band - mit einer medienanthropologischen Perspektive, die er in seinem Vortrag unter dem Titel „D er Medien-M ensch in der Sicht einer Ä sthetischen Theologie" vorstellte.

In seinem innovativen, anregenden Ansatz versteht Huizing die Theologie seit Paulus als eine erste „Schrift-Medien,Wissenschaft"', in der das Medium Schrift als „Eindrucksverstärker” des Lebens Jesu verwendet wird. Deshalb wird die Medien-

Ästhetik für ihn zu einer „Aufbauwissenschaft der Theologie". Umgekehrt hat die Theologie besondere Kompetenzen, die vielfältigen und oftmals sehr innovativen und anregenden Neuinszenierungen christlicher Überlieferungen in $\mathrm{Ki}$ nofilmen, Videoclips und anderen Medien zu entdecken. Über eine solche Kultur- und Medienhermeneutik hinaus geht es Huizing allerdings auch um eine theologische Auseinanderset- zung, die sowohl in positiver Aufnahme der Medieninhalte als auch in abgrenzender Kritik der in Medienerzählungen häufig propagierten „stoischen Distanzgestik des Cool eine christliche Liebesgestik der Toleranz und Solidarität" gegenüber stellt.

Prof. D r. D ieter Spanhel, Erziehungswissenschaftler von der Universität E rlangen-Nürnberg, fragte in seinem Vortrag nach der „Bedeutung anthropologischer bzw. kulturanthropologischer Aspekte für die Medienpädagogik". Nach seiner Auffassung ist die anthropologische Betrachtungsweise für die Medienpädagogik von einer grundlegenden Bedeutung, wobei gleichermaßen wichtig ist, dass die verschiedenen Einzeldisziplinen, die sich mit Medien beschäftigen, ihre jeweilige Perspektive in eine Anthropologie der Medien einbringen.

Die Medienpädagogik hat sich in Spanhels Sicht bisher viel zu kurzatmig mit Fragen des Beziehungsverhältnisses zwischen Mensch und $\mathrm{Me}$ dien befasst (Was machen die Medien mit den Menschen? - Was macht der Mensch mit den Medien?). Dabei wurde die anthropologische Dimension, die sich aus den Funktionen des Zeichengebrauchs in den anthropologischen Grundverhältnissen des Menschen zur Welt, zum Mitmenschen und zu sich selbst ergibt, weitgehend außer acht gelassen. So ist bisher nach seiner Einschätzung die Medienpädagogik noch nicht zu einer klar begründeten Beschreibung ihres Gegenstandsbereichs und zu einer systematischen Ausdifferenzierung ihrer Fragestellungen gelangt.

Eine zentrale anthropologische Fundierung der Medienpädagogik sieht Spanhel in der Tatsache, dass $\mathrm{Me}$ dienentwicklung (im weitesten Sinnn) nur durch Lernen und Erziehen möglich ist: Der Mensch muss auf der anthropologischen Grundlage der Fähigkeit zum Zeichengebrauch die Kompetenzen selbstständig ausbilden, die es ihm ermöglichen, die Chancen der Medien für ein neues Menschsein zu nutzen und ihre Gefahren und Probleme zu überwinden, und dabei ist er auf vielfältige pädagogische Hilfestellungen angewiesen. Auf der anderen Seite müssen sich laut Spanhel die Erziehungswissenschaft und die Medienpädagogik der medialen Konstruiertheit aller pädagogischen Prozesse bewusst sein. Im Zusammenhang mit den rasanten Medienentwicklungen müssen sie sich daher verstärkt den Veränderungen in dem anthropologischen 
Grundverhältnis Mensch - Medien zuwenden und der Frage, wie sich damit die Basis allen pädagogischen Handelns verändert.

Den Abschluss des ersten Tages bildete ein kreatives, sinnlich-mediales Performance-Projekt, das die Theaterpädagogin Gabriele Czerny und die Literaturdidaktikerin Prof. Dr. Gudrun MarciBoehncke (beide von der PH Ludwigsburg) zusammen mit Studierenden erarbeitet hatten. Unter dem Titel „Messenger - Moments of Man and Media" wurde in z. T. heiteren, z. T. beeindruckend dichten und impulshaltigen Dialogen, Sketchen, Tanz- und Musik-Sequenzen das Tagungsthema auf eine wohltuend alternative und gerade deshalb auch Denkprozesse in Gang setzende Art präsentiert.

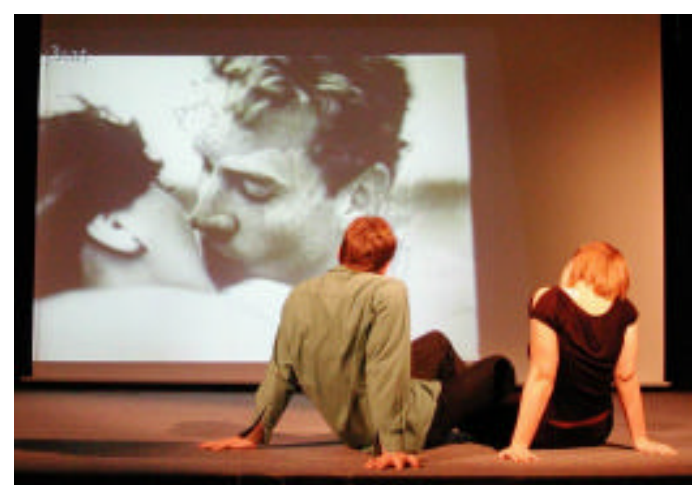

Szenenfoto aus dem Performance-Projekt „Messenger - Moments of Man and Media”

Der zweite Tag des Symposiums war geprägt von drei Vorträgen der "Lokalmatadoren” der PH Ludwigsburg. Der Literaturwissenschaftler $\mathrm{D} \mathrm{r}$. Boy $\mathrm{H}$ inrichs widmete sich mit einem aktuellen, hochinteressanten Thema: „Körper-Kult und KultKörper. Zum M enschenbild in populärkulturellen TV Formaten". Ausgangspunkt ist für Hinrichs die lebensweltliche D ominanz des Körpers, wie sie sich in den Schlagworten "Körper-Kult", „Fitness”, „Wellness” u. a. niederschlägt, wie sie sich aber auch in der immer bewussteren und absichtsvollen Kreierung von „Kult-Körpern“ in den Medien und darüber hinaus zeigt. Für heutige Durchschnitts-Jugendliche ist beispielsweise längst selbstverständlich: „Körper machen Leute"; der Körper selbst wird zum modellierbaren Kultur-Produkt, den man nach Gusto (v. a. der Peer-G roup) stylt.

Vor diesem Hintergrund und mit einer durch die Cultural Studies geprägten Perspektive analysierte Hinrichs dann die Inszenierung der Körper in den TV Soap Operas. In Serien wie „Gute Zeiten, schlechte Zeiten" (GZSZ) entdeckt er als wesentlichen Aspekt das Vorliegen einer MetaInszenierung: inszeniert wird, wie ein Körper inszeniert wird. Die Kult-Körper inszenieren Körper-Kult in szenischer Konkretion. Im Kreislauf von Produktion, Präsentation und Rezepti- on - so lässt sich Hinrichs Fazit knapp zusammenfassen - entwickelt sich das popularkulturelle Phänomen des Körper-Kults in höchst ambivalenter Weise (weiter).

Der Philosoph und Ethiker Prof. Dr. Matthias Rath lieferte mit seinem Referat „D er $\mathrm{H}$ omo M edialis und seine Brüder - zu den G renzen eines anthropolocischen W esensbegriffs" einen erhellenden Beitrag $\mathrm{zu}$ den Vor- und Grundfragen einer Medienanthropologie. In Auseinandersetzung mit zentralen klassischen Konzepten der Anthropologie war es seine Absicht, solche Aspekte herauszufiltern, die für eine (Medien-)Ethik sinnvoll und fruchtbar sein können.

Als besonders wirkmächtig haben sich in seiner Sicht die Konzepte des homo oeconomiaus und des homo sociologicus erwiesen. Gerade sie stellen jedoch den Menschen in besonderer Schärfe vor die Frage, ob und wie die anthropologischethische Grundbestimmung des Menschen als Person und als Wesen, das eine eigene Identität „,besitzt” angesichts der offensichtlichen sozioökonomischen Fremdbestimmung des Menschen durchgehalten werden kann. Zentral ist für Rath in diesem Zusammenhang die Einsicht, dass der Mensch über ein „Selbstverständnis” verfügt, also über die hermeneutische Fähigkeit, sich selbst und seine Welt zu verstehen und auszulegen. Nach Ernst Cassirer kann und tut der Mensch dies immer im "Medium" symbolischer Formen. In einem gewissen aporetischen Zirkelschluss lässt sich somit sagen, dass jede Anthropologie - auch die Bestimmung des Menschen als animal symbolicum - selbst ein symbolisches (selbstreferentielles) Konstrukt des Menschen ist. Gerade hinter diese Einsicht aber gibt es kein Zurück mehr, womit sowohl die „Tiefe” als auch die Grenzen einer Medienanthropologie deutlich werden.

Mit der Unterhaltung als einer für die gegenwärtige Medienkultur zentrale Kategorie beschäftigte sich der evangelische Theologe Prof. D r. M anfred L . Pirner in seinem die Tagung abschließenden Vortrag mit dem Titel „Muss denn Unterhaltung Sünde sein?' - Anthropologische Grundlagen der Medienunterhaltung". Seine Ausgangsthese (unter der Überschrift "Schon Adam und Eva haben sich gut unterhalten"): E rst ein anthropologisch und kulturtheoretisch grundge legter U nterhaltungsbegriff ermöglicht eine qualifizierte und differenzierte Beurteilung und Kritik der M edienunterhaltung, weil so U nterhaltung sowohl von den G rundbedürfnissen des Menschen als auch von ihrer kulturellen Bedingtheit her verstanden wird.

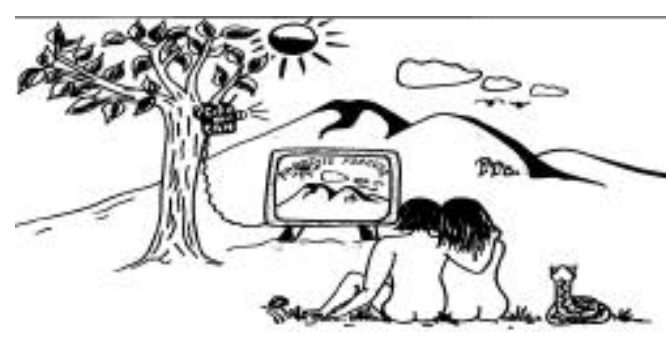


Theologische profilierte Aspekte eines solchen Unterhaltungsbegriffs versuchte Pirner im Rückgriff auf Thomas von Aquin (Unterhaltung als entlastetes, vergnügliches Selbst-E rleben) sowie durch eine eigene Interpretation des christlichen Rechtfertigungsglaubens zu entfalten: Gott sorgt für Unterhaltung, weil er - nach christlichem Glauben den Unterhalt des menschlichen Lebens garantiert, auch in Leid und Tod. Das dadurch ermöglichte entlastete Leben kann als Ermöglichungsgrund für Unterhaltung betrachtet werden: Das Vertrauen auf diesen LebensUnterhalt (Gottes) wird zur Basis für ein unterhaltsames Leben. Umgekehrt folgert Pimer: In jeder Unterhaltung, also in jedem vergnüglich-humorvollen Tun des Menschen schwingt unausgesprochen das Grundvertrauen mit, dass der eigene Lebens-Unterhalt garantiert ist, anders formuliert: dass das Leben sinnvoll und vertrauenswürdig ist - dies gilt auch für nicht-religiöse Menschen.

Unter den Überschriften „Unterhaltung ist das säkularisierte "Evangelium" in einer Welt des "Gesetzes", "Unterhaltung ist, wie die Religion, ein Übergangsphänomen" und "Schon Adam und Eva haben erfahren: Unterhaltung kann auch Sünde sein" wurden die genannten Grundlagen weiter entfaltet und ergänzt.

\section{Medienanthropologie - wie geht es weiter?}

Die etwa dreißig Tagungsteilnehmer/innen waren sich einig: $\mathrm{D}$ as bei der Tagung begonnene $G$ espräch soll weiter gehen. Weitere Tagungen wurden ebenso angeregt wie die Einrichtung eines Internet-Forums zum Austausch und zur gegenseitigen Information.

Zunächst wird aber vor allem ein Sammelband die gehaltenen Vorträge in Druckform zur Verfügung stellen. Darüber hinaus werden weitere interessante Beiträge aufgenommen. Der Sammelband wird voraussichtlich im Herbst 2003 erscheinen. Die vorläufige Inhaltsübersicht sieht folgendermaßen aus:

\section{Grundlagen}

M anfred Fassler (Prof. D r., Kulturanthropologe und Soziologe, U niversität F rank furt/ $\mathrm{M}$ ain)

Die mediale Selbstbefähigung des Menschen

$\mathrm{H}$ ans-D ieter Kübler (Prof. D r., M edienwissenschaftler, $\mathrm{F}$ achhochschule $\mathrm{H}$ amburg)

Wie anthropologisch ist mediale Kommunikation? Über Sinn und Nutzen einer neuen Teildisziplin

M atthias Rath (Prof. D r., Philosoph und M edienethiker, PH L udwigsburg)

D er Homo Medialis und seine Brüder - zu den Grenzen eines anthropologischen $\mathrm{We}$ sensbegriffs

\section{Perspektiven}

Stefan A ufenanger (Prof. D r., E rziehungswissenschatter und $\mathrm{M}$ edienpädagoge, U niversität $\mathrm{H}$ amburg)

Invasion aus unserer Mitte. Perspektiven einer Medienanthropologie

D ieter Spanhel (Prof. D r., E rziehungswissenschaftler, U niversität E rlangen-N ürnberg)

Die Bedeutung anthropologischer bzw. kulturanthropologischer Aspekte für die Medienpädagogik

Manfred L. Pirner (Prof. D r., E v. Theologe, PH Ludwigsburg)

Die Medialität des Menschen. Theologische Aspekte zu einer Anthropologie der Medien

Klaas H uizing (Prof. D r., E v. Theologe und Schriftsteller, U niversität $W$ ürzburg)

Der Medien-Mensch in der Sicht einer Ästhetischen Theologie

\section{Konkretionen}

Boy H inrichs (D r., L iteraturwissenschaftler, Pädagogische $\mathrm{H}$ ochschule L udwigsburg)

Körper-Kult und Kult-Körper. Zum Menschenbild in populärkulturellen TV-Formaten

Christof E hrhart (D r., Politikwissenschaftler und $\mathrm{Me}$ dienberater, 0 berägeri/ Schweiz)

Handlungskalküle im Medienfeld. Rollenmodelle des medialen Menschen

G erd Buschmann (D r., E v. Theologe, PH L udwigsburg) D as Menschenbild der Werbung

Manfred L. Pirner (Prof. Dr., Ev. Theologe, PHL) Muss denn Unterhaltung Sünde sein? - Anthropologische G rundlagen der Medienunterhaltung und ihre medien- wie religionspädagogischen Konsequenzen

Gabriele Czerny (Theaterpädagogin, PH L) / G udrun Mard-Boehncke (Prof'in D r., L iteraturdidak tik erin, PH L )

„Messenger: Moments of Man and Media” - Ein Performance-Projekt der Abteilung D eutsch der Pädagogischen Hochschule Ludwigsburg

\section{Zusätzliche zugesagte Beiträge}

H orst N iesyto (zu II.) (Prof. D r., M edienpädagoge, Pädagogische $\mathrm{H}$ ochschule L udwigsburg)

W olfgang W unden (zu I.) (D r., Kath. Theologe und L eiter der A bt. U nternehmensstrategie des SW R, Stuttgart) 